\title{
Advances of systemic treatment for adult soft-tissue sarcoma
}

\author{
Wenshuai Liu ${ }^{1}$, Quan Jiang ${ }^{2}$, Yuhong Zhou ${ }^{3}$ \\ ${ }^{1}$ Department of General Surgery, Shanghai Public Health Clinical Center, Zhongshan Hospital (South Branch), Fudan University, Shanghai 200032, \\ China; ${ }^{2}$ Department of General Surgery, ${ }^{3}$ Department of Medical Oncology, Zhongshan Hospital, Fudan University, Shanghai 200032, China \\ Contributions: (I) Conception and design: Y Zhou; (II) Administrative support: Y Zhou; (III) Provision of study materials or patients: W Liu; (IV) \\ Collection and assembly of data: W Liu; (V) Data analysis and interpretation: W Liu; (VI) Manuscript writing: All authors; (VII) Final approval of \\ manuscript: All authors. \\ Correspondence to: Yuhong Zhou. Zhongshan Hospital, 180 Fenglin Road, Shanghai 200032, China. Email: zhou.yuhong@zs-hospital.sh.cn.
}

\begin{abstract}
Soft-tissue sarcoma (STS) is a group of rare but highly heterogeneous neoplasms. Systemic treatment with cytotoxic chemotherapy and targeted agents is one of the main therapeutic modalities in patients with unresectable or metastatic disease, while adjuvant and neoadjuvant chemotherapy for adulttype sarcomas remain controversial. Although an anthracycline (doxorubicin) and ifosfamide remain the cornerstone for chemotherapy, advances have been made recently to exceed its limited efficacy, other agents such as trabectedin, eribulin have been approved. In a recent study, the addition of platelet-derived growth factor receptor (PDGFR) antibody-olaratumab to doxorubicin resulted in prolongation of progression-free survival and overall survival, which really means a breakthrough for STS. There is more emerging evidence of different sensitivity to treatment for different histological subtypes, second-line treatment for advanced sarcoma is being increasingly driven by histology. Cytotoxic drugs such as dacarbazine, gemcitabine, and taxanes have shown moderate activity in specific subtypes. Tyrosine kinase inhibitors (TKIs), including pazopanib and anlotinib, appear to be the promising targeted therapies. Other signal pathway inhibitors as CDK4/CDK6 inhibitor, imatinib, mTOR inhibitors, ALK inhibitor has shown some preliminary effect that need to be verified in the future trials. Checkpoint inhibitors as anti-PD-1 and CTLA-4 monoclonal antibodies have been used as a single agent or in combination in the early clinical trials, while further research needs to focus on better patient selection and new combinational strategies. In this review, we aim to summarize the advances of chemotherapy, targeted therapy and immunotherapy in the management of STS.
\end{abstract}

Keywords: Sarcoma; advanced sarcoma; metastatic sarcoma; chemotherapy; targeted therapy; checkpoint inhibitor

Submitted Jun 15, 2018. Accepted for publication Aug 06, 2018.

doi: $10.21037 /$ cco.2018.08.02

View this article at: http://dx.doi.org/10.21037/cco.2018.08.02

\section{Introduction}

Soft-tissue sarcomas (STS) are a group of rare but highly heterogeneous neoplasms with mesenchymal origin. The curative management for localized disease is surgical resection, combined with or without radiotherapy (RT) for selected patients. Systemic treatment with cytotoxic chemotherapy and molecular targeted agents is one of the main therapeutic modalities in patients with advanced or metastatic disease. While adjuvant and neoadjuvant chemotherapy for pediatric sarcoma such as rhabdomyosarcomas, Ewing sarcomas and osteosarcomas is established, it's role in adult-type sarcomas remains controversial. With the increasing knowledge in the molecular basis of pathogenesis of sarcoma and emerging of novel drugs, the treatment outcomes for patients with STS will improve greatly in the future. In this review, we aim to summarize the advances of chemotherapy, targeted therapy and immunotherapy in the management of STS. 


\section{Adjuvant chemotherapy for STS}

There is an urgent need to determine the role of adjuvant chemotherapy for STS since up to half of high-risk patients will eventually relapse or develop distant metastasis. Over 20 studies on adjuvant therapy for STS have been performed. Unfortunately, these trials reported conflicting data. Most of them have been small, enrolled different risk patients and treated with different chemotherapy regimens. Two meta-analyses conducted on the randomized controlled trials regarding adjuvant chemotherapy for STS by Sarcoma Meta-analysis Collaboration (SMAC) have further explored the potential benefit of adjuvant chemotherapy for resected STS in adults. The first meta-analysis was published in 1997 including 14 studies which involved 1,568 adults with postoperative STS (extremities and others) to receive or not receive adjuvant doxorubicin-containing chemotherapy (1). After a median follow-up of 9.4 years, it was demonstrated a significantly lower risk for relapse, either local or distant metastatic, in chemotherapy arm than in observation arm, but the overall survival (OS) was not statistically significant although there was a trend towards improved [hazard ratio (HR) for death $0.89,95 \%$ confidence interval (CI): $0.76-1.03]$. Five recent studies using ifosfamide as part of adjuvant therapy were added to the SMAC updated meta-analysis in 2008 (2). The odds ratios (OR) for local recurrence was 0.73 (95\% CI: 0.56-0.94; $\mathrm{P}=0.02$ ), for distant and overall recurrence was 0.67 (95\% CI: 0.560.82; $\mathrm{P}=0.0001$ ), all in favor of chemotherapy, consistent with those found in the earlier meta-analysis. In terms of survival, doxorubicin alone had an OR of 0.84 (95\% CI: 0.68-1.03; $\mathrm{P}=0.09$ ), which was not statistically significant. However, when doxorubicin combined with ifosfamide, it can translate into a gain in OS $(\mathrm{OR} 0.77, \mathrm{P}=0.01)$ and absolute risk reduction of death of $6 \%$, which implies the role of ifosfamide in the adjuvant treatment of sarcomas.

The first large study to incorporate ifosfamide as part of adjuvant therapy for STS is from the Italian Sarcoma Study Group which was designed on relatively restricted enrollment criteria (3). A total of 104 highrisk postoperative patients (grade 3-4, primary diameter $\geq 5 \mathrm{~cm}$ or any size recurrent tumor) in extremities or girdles were randomized to the dose intensive chemotherapy group $\left(5\right.$ cycles of epirubicin $60 \mathrm{mg} / \mathrm{m}^{2}$ over 2 days and ifosfamide $1.8 \mathrm{~g} / \mathrm{m}^{2}$ over 5 days) or control group. After a median follow-up of 59 months, median disease-free survival (DFS) (48 vs. 16 months) and median OS (75 vs. 46 months) were significantly better in the chemotherapy arm. These data imply a survival advantage of high-dose intensified chemotherapy for patients with high-risk extremity STS.

Although there was a significant survival benefit for the trial of the Italian Sarcoma Group, no difference in the recurrence-free survival (RFS) or OS were demonstrated in the European Organization for Research and Treatment of Cancer (EORTC) trials. The largest phase III randomized study of adjuvant chemotherapy was EORTC-62931 (4). A total of 351 grade 2-3 completely resected patients were recruited to 5 cycles of doxorubicin $75 \mathrm{mg} / \mathrm{m}^{2}$ and ifosfamide $5 \mathrm{~g} / \mathrm{m}^{2}$ per cycle versus observation. However, this study was negative which showed no statistically significant difference in terms of DFS or OS. The estimated 5 -year RFS was 52\% in both arms and OS was $69 \%$ (control arm) and 64\% (chemotherapy arm), respectively. To explain the difference between Italian Sarcoma Study Group and EORTC 62931 is the latter study included a heterogeneous group of high and low risk patients (67\% extremity tumors, $60 \%$ high-grade, $40 \% \geq 10 \mathrm{~cm}$ ) and its suboptimal dosage of ifosfamide. In another earlier large EORTC study, postoperative 486 patients were randomized to combination chemotherapy with cyclophosphamide, vincristine, doxorubicin, and dacarbazine (CyVADIC) for 8 cycles or observation (5). While DFS and local control were both better in the chemotherapy arm, OS was not significantly different between the two arms.

Even in the prospective randomized trials, the role of adjuvant chemotherapy cannot be defined if the population of patients is unselected. Most trials have involved relatively small patient population, with heterogenous groups of recurrence risk. The criteria used to select patients for adjuvant chemotherapy should be based upon who will truly benefit. How to define high-risk patients depends on several factors, including tumor grade, size, histological type, primary location and the quality of surgery. However, there is no universally accepted definition for high-risk patients in STS. The most widely accepted grading system is proposed by the Sarcoma Group of the French Federation of Cancer Centers (FNCLCC). Tumors are classified according to three parameters: the mitotic index, the presence of necrosis, and cell differentiation. This classification has demonstrated prognostic value, with 5 -year survival rates of $95 \%, 75 \%$, and $45 \%$ in patients with grade 1,2 , or 3 tumors, respectively (6).

A recent pooled analysis of the above mentioned two large EORTC studies aimed to evaluate whether adjuvant chemotherapy benefited any particular subgroup 
patients (7). However, RFS and OS were only improved in the $\mathrm{R} 1$ resection group. It failed to demonstrate any other factors (size, histology, grade) as predictors of improved survival on multivariate analysis.

Adjuvant chemotherapy has failed so far to consistently improve OS. The reason for that may be the criteria now used to select patients for adjuvant treatment (usually high grade, primary tumor $\geq 5 \mathrm{~cm}$, deeply seated or locally recurrent extremity sarcomas) are not optimal. We need a clear risk stratification for patient selection for adjuvant therapy. Several prognostic nomograms have been developed to predict OS and risk of distant metastasis in patients undergoing resection of STS of the extremities, but all of them have some limitations $(8,9)$. In the future, clinical design for adjuvant chemotherapy should incorporate these tools for accurate risk stratification.

Moreover, it should be recognized that chemosensitivity is another important factor for consideration in selection patients for adjuvant therapy. It is well known from studies in the metastatic sarcomas that myxoid/round cell liposarcomas and synovial sarcomas are relatively chemosensitive subtypes of STS.

To make some sense of who will be given adjuvant therapy, it is useful to bear in mind that it needs to be discussed on an individual case by case basis, taking into consideration site of disease (extremity/retroperitoneal/ trunk), size, grade, histological subtypes, chemosensitivities as well as PS of the patient, comorbidities, and age. Recently, a mobile device called "Sarculator" which may predict 10-year probability of OS and incidence of distant metastasis in STS patients that can help us to identify highrisk STS patients who fit for adjuvant chemotherapy (10).

\section{Neoadjuvant chemotherapy for STS}

Neoadjuvant chemotherapy is already a well-adopted approach in the management of osteosarcoma. However, as the efficacy of chemotherapy for metastatic STS is relatively low, the role of neoadjuvant chemotherapy in adult STS is ill defined. There are many theoretical advantages for giving neoadjuvant chemotherapy before surgery, such as tumor cytoreduction for limb salvage surgery, elimination of micrometastases as well as prediction of the effectiveness of chemotherapy. However, in STS, there is lack of evidence specifically addressing the role of chemotherapy when used in a neoadjuvant setting. A European phase II trial enrolled 134 patients with resectable high-risk primary and recurrent STS (11). "High-risk" defined as tumors $>$ or $=8 \mathrm{~cm}$ of any grade, or grade II/III tumors $<8 \mathrm{~cm}$, or grade II/III locally recurrent tumors, or grade II/III tumors with inadequate surgery. These high-risk patients were randomized between either surgery alone or 3 cycles of doxorubicin $50 \mathrm{mg} / \mathrm{m}^{2}$ and ifosfamide $5 \mathrm{~g} / \mathrm{m}^{2}$ before surgery. At a median followup of 7.3 years, the 5 -year DFS is estimated at $52 \%$ for the no chemotherapy and $56 \%$ for the chemotherapy arm $(\mathrm{P}=0.3548)$. The 5 -year OS for both arms is $64 \%$ and $65 \%$, respectively $(\mathrm{P}=0.2204)$. Despite neoadjuvant chemotherapy with doxorubicin and ifosfamide at these doses and with this schedule was feasible and did not compromise subsequent treatment, it was not powered to draw definitive conclusions on benefit of neoadjuvant chemotherapy even in these selected high-risk patients. The low chemotherapy intensity used and patients with a large variety of histologies and varying chemosensitivities in this trial may potentially impact the possible benefit of neoadjuvant chemotherapy.

In light of the dose intense combination of ifosfamide $\left(9 \mathrm{~g} / \mathrm{m}^{2}\right)$ plus epirubicin $\left(120 \mathrm{mg} / \mathrm{m}^{2}\right)$ with G-CSF support may improve therapeutic efficacy, a multicenter international phase III study (12) was designed by Italian Sarcoma Group and the Spanish Sarcoma Group (ISG) to compare 3 cycles of preoperative full-dose epirubicin and ifosfamide ( $\operatorname{arm~A}$ ) with 5 cycles of the same drugs given perioperatively ( 3 neoadjuvant cycles followed by surgery and 2 further adjuvant cycles) (arm B). A total of 328 highrisk patients were recruited, with 164 patients in each arm. At a median follow-up of 63 months, 5-year OS probability was 0.70 for the entire group of patients (0.68 in arm A and 0.71 in arm B). The HR of arm A versus arm B was 1.00 (90\% CI: 0.72-1.39). It was concluded from this study that 3 cycles of full-dose preoperative chemotherapy were not inferior to 5 cycles of perioperative chemotherapy in terms of survival. In 2016, Gronchi et al. reported on their 10-year long-term follow-up of this trial (13). The non-inferiority of 3 cycles in comparison to five is confirmed. These results showed that if 3 cycles of full-dose neoadjuvant chemotherapy have been given, it does not need to give further adjuvant cycles. However, this study did not address whether these 3 cycles of full-dose chemotherapy given preoperative is superior or equal to postoperative with longterm survival.

Despite doxorubicin and ifosfamide are the two most active drugs in STS, there is more emerging evidence of different sensitivity to treatment for different histological subtypes. Promising activity has been reported from varying histology-specific regimens in treatment of metastatic STS. In a recently published phase III trial by ISG (ISG- 
STS-1001), 3 cycles of neoadjuvant histology-tailored chemotherapy were compared to standard chemotherapy with full dose epirubicin and ifosfamide (14). 287 highrisk patients with five different histological subtypes of STS were randomly assigned to standard chemotherapy or histology-tailored therapy (trabectedin for high-grade myxoid liposarcomas (MLPS) ( $\mathrm{n}=64)$, high-dose ifosfamide alone for synovial sarcoma $(n=70)$, etoposide plus ifosfamide for malignant peripheral nerve sheath tumors $(n=27)$, gemcitabine plus dacarbazine for leiomyosarcoma (LMS) $(\mathrm{n}=28)$, and gemcitabine plus docetaxel for undifferentiated pleomorphic sarcoma (UPS) ( $\mathrm{n}=97)$. The primary endpoint was DFS. With a median follow-up of 12.3 months, the projected DFS at 46 months was $62 \%$ in the standard chemotherapy group, better than $38 \%$ in the histotypetailored chemotherapy group (HR for DFS was 2.0, 95\% CI: 1.22-3.26; $\mathrm{P}=0.006$ ) across various histological subtypes, with the exception of high-grade MLPS, in which DFS was similar between trabectedin and standard chemotherapy. The OS probability at 46 months was 0.89 and 0.64 (log rank, $\mathrm{P}=0.003)$ in the standard and in the tailored arm, respectively. An absolute benefit of nearly $20 \%$ on PFS translates into an advantage on OS. Although neoadjuvant histotype-tailored chemotherapy regimen did not show any advantage over the standard chemotherapy regimen, the benefit with the standard chemotherapy regimen further confirmed the value of neoadjuvant chemotherapy itself in patients with high-risk STS.

From ESMO guideline, for STS, there is no consensus on the current role of adjuvant chemotherapy (15). It can be proposed as an option to the high-risk patient (high-grade, deep, $>5 \mathrm{~cm}$ tumor) for a shared decision-making with the patient or within clinical trials. Adjuvant chemotherapy is not used in histological subtypes known to be insensitive to chemotherapy. If the decision is made to use chemotherapy as upfront treatment, it may well be used preoperatively. From the study of ISG-STS-1001, a benefit may be gained when 3 cycles of full-dose anthracycline-ifosfamide regimen in selected high-risk STS of extremity/trunk wall.

\section{Chemotherapy in metastatic STS}

\section{First-line chemotherapy}

For the majority of patients with unresectable and metastatic disease, systemic therapy is administered with palliative intention. As in the adjuvant setting, anthracycline and ifosfamide remain the cornerstone for more than
30 years regardless of subtype. However, there are few studies directly assessed whether doxorubicin should be administered alone or in combination with ifosfamide. The randomized phase III EORTC 62012 trial was conducted, a total of 455 locally advanced or metastatic, grade 2 or 3 softtissue sarcoma patients were randomly assigned to receive either single-agent doxorubicin $\left(75 \mathrm{mg} / \mathrm{m}^{2}\right)$ or doxorubicin $\left(75 \mathrm{mg} / \mathrm{m}^{2}\right)$ with ifosfamide $\left(10 \mathrm{~g} / \mathrm{m}^{2}\right.$ over 4 days $)$ with growth factor support (16). Patients were treated every 3 weeks for a maximum of 6 cycles or until progression. At a median follow-up of 56 months, the difference of OS did not achieve statistical significance. Median OS was 14.3 months with the combination and 12.8 months with doxorubicin alone ( $\mathrm{HR}=0.83 ; \mathrm{P}=0.076)$. Median PFS, however, was 7.4 months with the combination and 4.6 months with doxorubicin alone, for a $26 \%$ reduction in risk that was statistically significant $(\mathrm{HR}=0.74 ; \mathrm{P}=0.003)$. The objective response rate (RR) was $26.5 \%$ with the combination and $13.6 \%$ with doxorubicin. Despite colonystimulating factor support, the most common grade 3 and 4 toxic effects were all more common in the combination than in the doxorubicin alone group: leucopenia (43\% vs. $18 \%$ ), neutropenia ( $42 \%$ vs. $37 \%$ ), febrile neutropenia ( $46 \%$ vs. $13 \%$ ), anemia ( $35 \%$ vs. $5 \%$ ), and thrombocytopenia ( $33 \%$ vs. $<1 \%$ ). The lack of OS advantage but with more toxicities for combination regimen do not support its routine use in the setting of advanced incurable disease unless there is an immediate need to decrease tumor bulk, improve symptoms or translate into resection.

Until now, the standard first-line treatment for STS has been doxorubicin. Is there any choice to consider when selecting first-line treatment beyond doxorubicin?

According to phase II and retrospective studies, the combination of gemcitabine and docetaxel may be effective in treating STS. The GeDDiS trial was a phase III, randomized, multicenter study to compare the combination of gemcitabine and docetaxel with doxorubicin in patients with previously untreated advanced unresectable or metastatic STS (17). Patients were randomly assigned to the control arm $\left(75 \mathrm{mg} / \mathrm{m}^{2}\right.$ of doxorubicin) or the investigational arm $\left(675 \mathrm{mg} / \mathrm{m}^{2}\right.$ of gemcitabine on days 1 and 8 plus $75 \mathrm{mg} / \mathrm{m}^{2}$ of docetaxel on day 8 every 21 days). A total of 257 patients were enrolled, the median followup was 19 months. The primary endpoint of 24-week PFS was identical between arms, $46 \%$ each. However, patients in the investigational arm had lower dose intensity ( $83.3 \%$ vs. $94.6 \%$ for doxorubicin), more dose delays ( $55.5 \%$ vs. $45.7 \%$ for doxorubicin), and more withdrawals 
because of unacceptable toxicity $(10.2 \%$ vs. $0.8 \%$ for doxorubicin). Moreover, no differences in efficacy were found between histology subtype groups, such as LMS or nonleiomyosarcoma. This result again confirmed that single agent doxorubicin should be the preferred first-line option, given greater tolerability and potentially favourable efficacy.

Although doxorubicin remains a backbone for sarcoma treatment, its $\mathrm{RR}$ is relatively low while with significant toxicities. As doses exceeding $75 \mathrm{mg} / \mathrm{m}^{2}$, doxorubicin is associated with cardiotoxicities, myelosuppression and mucositis. Several types of anthracycline have been recently tested in first-line treatment to improve its efficacy in STS. Aldoxorubicin is a novel albumin-binding prodrug of doxorubicin which contains a carboxylic hydrazone and covalently binds to albumin in the blood until reaching into tumor tissue, where the acidic microenvironment breaks the covalent bond with albumin and release doxorubicin. This allows for greater doses (3.5-4 times of the standard doxorubicin dose) of doxorubicin to be administered while reducing its side effects. An international multicenter phase $2 \mathrm{~b}$ randomized study has evaluated the efficacy and safety of aldoxorubicin $v s$. doxorubicin in patients with advanced STS (18). A total of 123 patients with histologically confirmed metastatic, locally advanced unresectable STS were randomized 2:1 to receive $350 \mathrm{mg} / \mathrm{m}^{2}$ aldoxorubicin $\left(260 \mathrm{mg} / \mathrm{m}^{2}\right.$ doxorubicin equivalents) intravenous (IV) or $75 \mathrm{mg} / \mathrm{m}^{2}$ doxorubicin every 3 weeks for up to 6 cycles. In this study, aldoxorubicin was associated with more than a doubling of both overall RR (ORR: 25\% vs. 0\%) and PFS (5.6 vs. 2.7 months; $\mathrm{P}=0.02$ ). A similar percentage of neutropenic fever between two arms (15\% vs. $16 \%)$, and a higher rate of decreased cardiac output was observed in doxorubicin arm (22\%) compared with aldoxorubicin arm (11\%). No patient treated with aldoxorubicin had ejection fractions below $50 \%$ versus $9.4 \%$ of patients that had received doxorubicin. Most importantly, there was no clinically significant reduction in cardiac function in the aldoxorubicin patients despite receiving high equivalent of doxorubicin dose. Aldoxorubicin represents a promising drug for treatment of sarcomas. The drug has minimal cardiac toxicity, which represents a significant advantage to doxorubicin. Preliminary phase 3 study results demonstrate PFS advantage in patients with LMS and liposarcoma. However, more studies are needed to establish the role of aldoxorubicin in sarcoma treatment.

Amrubicin is a third-generation anthracycline, which has been suggested to be less toxic than doxorubicin, especially for cardiac toxicity. A phase II multicenter single arm study was done to evaluate the efficacy and tolerability of amrubicin in advanced STS (19). A total of 24 patients were treated with amrubicin $40 \mathrm{mg} / \mathrm{m}^{2}$ for 3 days in 21 days cycles in first-line therapy. The best ORR was $13 \%$, median PFS was 5.8 months, and median OS was 26 months. Grade 3 to 4 toxicities of febrile neutropenia and anemia occurred in $21 \%$ of treated patients. There was no significant cardiac toxicity up to a cumulative dose of $4,800 \mathrm{mg} / \mathrm{m}^{2}$. One patient with metastatic MLPS with TLS-CHOP translocation had a durable response thus indicate further study is warranted in this subtype.

Similar to aldoxorubicin and amrubicin, newer fosfamides, namely evofosfamide and palifosfamide have not yet led to substantial progress. Two randomised phase III clinical trials named SARC021 (20) and PICASSO (21) have tested doxorubicin in combination with evofosfamide or palifosfamide, respectively. In these two studies, the combination arm produced higher RR but more toxicity. Moreover, same as EORTC 62012 trial, there were no OS or PFS in favour of the combination arm.

Until now, doxorubicin with or without ifosfamide remains the standard first-line chemotherapy for most of STS.

\section{Histology-based second-line chemotherapy}

There is no standard regimen in second line treatment for STS. Beside anthracyclines and ifosfamide, there are other drugs with moderate activity in this disease. As different subtypes may have different sensitivity to different cytotoxic agents, beyond the first line, the treatment for STS is being increasingly driven by histology. For example, there is evidence of efficacy of gemcitabine in LMS and angiosarcoma; dacarbazine in LMS and solitary fibrous tumors (SFT); trabectedin in LMS and liposarcomas, especially in MLPS; taxanes and gemcitabine in angiosarcoma, eribulin in liposarcoma, etc.

\section{Eribulin}

Eribulin mesylate is an antimitotic agent, which acts by inhibiting microtubules' growth. A phase 2 trial assessed the safety and efficacy of eribulin in pretreated STS 128 patients who received eribulin $1.4 \mathrm{mg} / \mathrm{m}^{2}$ over $2-5 \mathrm{~min}$ at days 1 and 8 for every 3 weeks. The subtypes including adipocytic sarcoma (37 patients), LMS (40 patients), synovial sarcoma (19 patients), and other sarcomas (32 patients). The result was positive for primary goal (3-month PFS), in the group of adipocytic sarcoma with $46.9 \%$ patients were progression 
free at 12 weeks, and LMS [3-month progression-free rate (PFR): 31.6\%] (22). Anther randomized phase 3 trial in 452 advanced pretreated adipocytic sarcoma and LMS patients, $50 \%$ of patients received eribulin $\left(1.4 \mathrm{mg} / \mathrm{m}^{2}\right.$, intravenous on days 1 and 8$)$, the left over received dacarbazine (850$1,200 \mathrm{mg} / \mathrm{m}^{2}$, IV on day 1) for every 21 days (23). The median OS for eribulin and dacarbazine was 13.5 and 11.5 months, respectively (HR $=0.768,95 \%$ CI: 0.618-0.954; $\mathrm{P}=0.017)$. The $\mathrm{OS}$ was significantly improved (15.6 vs. 8.4 months) in liposarcoma cohorts, while no sense was seen in LMS. Based on these results, the FDA approved the using of eribulin in advanced pretreated liposarcoma in 2016 (http://www.fda.gov/.fda.gov). The most common grade 3-4 adverse events were neutropenia, anemia, fatigue, and febrile neutropenia.

\section{Trabectedin}

Trabectedin, derived from the marine ascidian, Ecteinascidia turbinata, is a natural alkaloid with multiple complex mechanisms of effects. Trabectedin, mainly binds to the minor groove of DNA and binds to guanine in N2 position, different from traditional alkylating agents which binds to the major groove of DNA and predominantly form crosslink to guanine in the N7 or O6 position (24). Trabectedin disturbs tumor cell cycle progression by retarding S-phase progression and inducing G2/M arrest (25).

In 2001, Delaloge et al. first reported the activity of trabectedin in 29 advanced STS patients who had failed in prior doxorubicin-based chemotherapy (26). In this investigation, there were $34.5 \%(10 / 29)$ stable disease (SD) sustaining more than 2 months and median time to progression of 2.8 months, $13.8 \%$ (4/29) PR, 6.9\% (2/29) minor responses with shrinking at least $30 \%$ in size. A late study recommended the dosage of trabectedin to be $1.5 \mathrm{mg} / \mathrm{m}^{2}$ as $24-\mathrm{H}$ continuous intravenous infusion (CIV) once every 3 weeks (27). Morgan et al. (28) compared $580 \mu \mathrm{g} / \mathrm{m}^{2}$ per week schedule with the "standard" $1.5 \mathrm{mg} / \mathrm{m}^{2} 24-\mathrm{H}$ CIV every 3 weeks schedule, the results indicated that every 3 weeks schedule superior to per week in median PFS (3.3 vs. 2.3 months; $\mathrm{P}=0.0418$ ).

Several phase 2 clinical trials have been conducted to verify the efficacy of trabectedin alone or combine with other chemotherapy drugs in advanced STS patients who fielded in previous first-line chemotherapy (29-32). In a retrospective study (31), 885 advanced STS patients received the "standard" q3 weeks regime, and 227 of them continued trabectedin up to disease progression. The longer trabectedin treatment until disease progression is associated with a significantly improved PFS (11.7 vs. 4.4 months) and OS (24.9 vs. 12.2 months). In an expanded access program for 1,895 advanced STS following failure of prior chemotherapy, suggested the patients with LMS and liposarcoma had significantly longer OS (16.2 vs. 8.4 months, respectively) compared to all other histological subtypes, as well as higher RR (6.9\% vs. 4\%, respectively). Several trials tried to verified the efficacy of trabectedin in specific STS subtypes, mainly concentrated on LMS and MLPS (33-36).

In 2015, Demetri et al. launched critical randomized phase 3 study using trabectedin $(\mathrm{n}=345) v s$. dacarbazine $(n=173)$ in locally advanced, unresectable or metastatic LMS and liposarcoma (37). In the trabectedin arm, trabectedin $\left(1.5 \mathrm{mg} / \mathrm{m}^{2}\right)$ was administered as a $24-\mathrm{H}$ CIV on day 1 of every 3 weeks, whereas in the dacarbazine arm, dacarbazine $\left(1 \mathrm{~g} / \mathrm{m}^{2}\right)$ was administered by intravenous infusion over 20-120 min on day 1 of every 21 -day cycle. OS was the primary end-point, and PFS was a secondary end-point. Fortunately, treatment with trabectedin resulted in a statistically significant improvement in PFS, with a PFS of 4.2 months and 1.5 months for trabectedin and dacarbazine, respectively ( $\mathrm{HR}=0.55 ; 95 \% \mathrm{CI}: 0.44-0.70 ; \mathrm{P}<0.001$ ). However, the primary end-point, interim analysis indicated no improvement in OS ( $\mathrm{HR}=0.87 ; \mathrm{P}=0.37$ ). Based on these dates, the United States Food and Drug Administration (FDA) approved the trabectedin for the treatment of unresectable or metastatic liposarcoma or LMS that has failed a prior anthracycline-containing regimen.

MLPS is, characteristically associated with $\mathrm{t}(12 ; 16)$ FUSCHOP translocation which accounting for $90 \%$ variants of MLPS, a STS subtype which is particularly sensitive to trabectedin. The chimeric product of this translocation modulates the activity of the C/EBP pathway, which is implicated in G1-S phase cell cycle progression, growth arrest, apoptosis, and developmental programs (38). Some of transcription play a crucial role in adipocytic differentiation, the trabectedin may interfere with the activity of modulation and induce expression or accumulation of some proteins which associated with terminal adipocytic differentiation. While in Europe, the drug is approved for all STS, and it brought the experiences in treatment of other subtypes of STS. In a retrospect study of the using trabectedin in 61 patients with synovial sarcoma, 9 of them had a partial response (PR), and 21 of them had SD (39).

Although trabectedin brings new hope for specific subtypes of STS, the side effects also have to be taken 
into consideration. In previous investigation (37), the most common adverse reactions are nausea (75\%), fatigue (69\%), vomiting (46\%), constipation and decreased appetite (37\%), diarrhea (35\%). Less common adverse reactions were dyspnea and headache (25\%), arthralgia and insomnia (15\%), and myalgia (12\%). The grade 3-4 side effects were, less than $10 \%$, neutropenia, thrombocytopenia, anemia, elevated liver enzymes. Thus, an important method in reducing toxicity is the use of dexamethasone prophylaxis $4 \mathrm{mg}$ po BID the day before the trabectedin CIV, and markedly decreased the grade 3-4 advanced effects $(\mathrm{P}=0.0001)(40,41)$. At the same time, don't forget to follow up blood biochemistry during trabectedin infusion.

With the efficacy of trabectedin and doxorubicin has firmly established in advanced STS, does the two drugs powerful combination will bring new hope for metastatic STS? In 2001, Takahashi and colleagues reported that the combination appeared to result in synergistic cytotoxicity in a sarcoma cell line. The trabectedin 24-H CIV prior to doxorubicin seemed to enhance the cytotoxic activity of the combination (42). Blay et al. studied a $60-\mathrm{mg}$ dose of doxorubicin administered as a bolus with subsequent 3-H CIV of trabectedin. The results indicated that $83 \%$ of patients had SD, $12 \%$ of them had PR, but all patients presented dosing limiting neutropenia, and all need hematopoietic growth factor support, and the maximum tolerated dose was doxorubicin $60 \mathrm{mg} / \mathrm{m}^{2}$ with trabectedin $1.1 \mathrm{mg} / \mathrm{m}^{2}$ (43). Another nonrandomized phase II study of the combination of trabectedin and doxorubicin in soft tissue and uterine LMS adopted the dosing the same with Blay et al. $59.6 \%$ patients with PR and $27.7 \%$ with PR in the uterine LMS cohort, while $36.1 \%$ patients with PR and $52.6 \%$ having SD in the soft-tissue LMS cohort. The median PFS (8.2 vs. 12.9 months) and median OS (20.2 vs. 35.5 months) for uterine LMS and soft-tissue LMS respectively. Although lacking the date about the outcomes of patients with soft-tissue LMS, the authors still pointed out that the RR, PFS and OS in the uterine LMS cohort did compare favorably to other uterine LMS cohorts treated with combinations like gemcitabine and docetaxel or doxorubicin and ifosfamide (44-46). In a randomized phase II study compared the doxorubicin $75 \mathrm{mg} / \mathrm{m}^{2}$ single agents with the combination of doxorubicin $60 \mathrm{mg} / \mathrm{m}^{2}$ and trabectedin $1.1 \mathrm{mg} / \mathrm{m}^{2}$ in a broader range of STS. Frustratedly, the study was stopped early because of the worsening expected PFS in experimental arm (47). The PFS in experimental arm and the control group was $5.5 \mathrm{vs}$. 5.8 months respectively. The second endpoints, RR and OS, were also no sense.

Above all, trabectedin is one of effective alternative chemotherapeutic drugs for unresectable or metastatic STS, especially in liposarcoma or LMS. If trabectedin is used in combination with other agents, special attention should be paid to the toxic reaction and the dosage of the drug, and at the same time take appropriately methods to reduce and monitor the toxicity.

\section{Others}

Both temozolomide and dacarbazine (DTIC), are alkylating agents, with temozolomide being a prodrug of dacarbazine which interferes with the biosynthesis of purine, showed activity in monotherapy in pretreated STS (48). In a prolonged schedule of temozolomide $\left(75-100 \mathrm{mg} / \mathrm{m}^{2}\right.$ per day during 6 consecutive weeks) in 48 pretreated STS patients, the 3 -month PFR was $39.5 \%$ and RECIST RR was $15.5 \%$, and the efficacy lasting for a long time (median of 12.5 months) in responding patients (49). Another study indicated modest activity in pretreated STS, and the LMS group had a median PFS and OS of 3.9 and 30.8 months, respectively (50). These drugs could be especially interesting in LMS. What's more, SFT also seems to benefit from dacarbazine and temozolomide based regimens $(51,52)$

The combination of dacarbazine and doxorubicin is one of the oldest drugs to have shown efficacy in STS. Primarily, DTIC was used as a monotherapy in advanced STS, and had a RR of $18 \%$; but a short time to progression (53). A study compared doxorubicin alone with the combination of doxorubicin and DTIC, showed increase in RR in advanced STS (54). In 2013, a systematic review investigated the using of DTIC as second line therapy with and without gemcitabine (55). The results indicated the combination had a high rate of disease free progression at 3 months (54.2\% vs. $35.2 \%$, respectively).

Paclitaxel has widely used in tumor chemotherapy and also shown activity in STS. In a phase 2 trial conducted by French sarcoma group, 30 advanced angiosarcoma patients were treated with weekly paclitaxel $80 \mathrm{mg} / \mathrm{m}^{2}$ days 1,8 , and the results showed that the 4 -month PFS reached $45 \%$. The same group also launched a combination trial of bevacizumab and paclitaxel, but failed to show the superiority to weekly paclitaxel (56). Another clinical trial also showed the activity in Kaposi sarcoma (57).

Thus, when we carry out chemotherapy regiments for STS, we'd better take the subtype, location, histological 
grade, tumor size, surgical margin, tumor metastasis, and previous treatment into consideration. In addition, patient's factors should not be ignored, factors including, but not limited to, patients' willingness, physical status, potential toxic risks related to the treatment, as well as possible quality-of-life impairments. Therefore, a multi-disciplinary team discussion is essential for all advanced STS in order to propose appropriate strategies and maximize the therapeutic effects.

\section{Targeted therapy}

Because of the heterogeneity of STS and the lack of driver mutations, the development of targeted therapy is lagging. However, the investigations of sarcoma genomics and mutations of signaling pathway have revealed several candidates for targeted therapy, and the angiogenetic pathway was found to be one of the promising targets (58).

\section{Tyrosine kinase inbibitors (TKIs) targeting angiogenesis}

Pazopanib is a potent and selective multi-targeted receptor tyrosine kinase (RTK) inhibitor that blocks tumour growth and inhibits angiogenesis. In the phase II EORTC 62043 study, patients were enrolled in four cohorts: LMS, liposarcoma, synovial sarcoma, and other histologies (59). The primary end-point was the PFR at 12 weeks, and the outcomes were evaluated in each cohort; 18 of 41 (44\%) patients in LMS cohort, 18 of 37 (49\%) patients in synovial sarcoma cohort, 16 of 41 (39\%) patients in other histologies cohort reached the progression-free at 12 weeks. However, liposarcoma cohort was stopped because of only 5 of 19 (26\%) patients reached the progression-free at 12 weeks. As a result, liposarcoma was excluded from the phase III study (PALETTE), in which median PFS was 4.6 months for the pazopanib-treated patients compared to 1.6 months for the placebo-treated patients $(\mathrm{P}<0.0001)(60)$. The results of the PALETTE study led pazopanib approved by the FDA in 2012.

Anlotinib is another RTK inhibitor, targeting VEGFR, FGFR, PDGFR, C-kit, etc. $(61,62)$. Anlotinib has shown single agent activity in a single arm, phase II study presented orally at 2016 ASCO. A randomized, doubleblind, placebo-controlled phase IIb trial was aimed at confirming the efficacy of anlotinib in treating STS (63). Recurrent advanced STS progressed after anthracyclinecontained therapies were randomized 2:1 to receive anlotinib or placebo. A total of 233 patients were enrolled in this trial. The primary endpoint was PFS and secondary endpoints were ORR, disease control rate (DCR) and OS. Median PFS was 6.27 months in the anlotinib arm versus 1.47 months in the control arm, the difference was very significant and the risk of disease progression was reduced by $67 \%$. Moreover, ORR was $10.13 \%$ versus $1.33 \%$, DCR was $55.7 \%$ versus $22.67 \%$, respectively.

Other small molecular TKI targeting angiogenesis including sunitinib, sorafenib, regorafenib, cediranib and apatinib have shown moderate activity in LMS, synovial sarcoma, alveolar soft part sarcomas, solitary fibrous tumours and angiosarcomas in small sample, phase II trials. The selection should be based on histologic subtype, patient characteristics, toxicity profile and accessibility of the drug.

\section{CDK4/CDK6 inbibitor}

CDK4 has been regarded as another promising target in the treatment of STS. Overexpression of the protein in more than $90 \%$ of well-differentiated/dedifferentiated liposarcoma (WDLS/DDLS) has been found (64). Palbociclib is a CDK4/CDK6-inhibitor, in a phase 2 study, it was shown that a 12 -week PFS rate of $66 \%$ in CDK4positive WDLS/DDLS (65). The previous phase 2 study has already proved the favorable of PFS at the 200-mg dose (for 14 days, every 3 weeks). Another study of palbociclib in 60 advanced WDLS/DDLS (125 mg daily for 21 days in 28 -day cycles), the results show that the 12-week PFS was 57.2 and median PFS was 17.9 weeks (66).

\section{Other TKIs}

With regard to other TKIs, imatinib is effective in dermatofibrosarcoma, mammalian target of rapamycin (mTOR) inhibitors are active in a proportion of PEComas (perivascular epithelioid cell tumours) and crizotinib in ALK-rearranged inflammatory myofibroblastic tumours.

\section{Monoclonal antibodies: olaratumab}

A possible breakthrough in the treatment of STS is represented by the recently published results of an open-label phase Ib/II trial comparing olaratumab and doxorubicin versus doxorubicin alone for firstline treatment of STS patients (67). Olaratumab is a recombinant monoclonal antibody that targets PDGFR $\alpha$, blocking PDGF-AA, PDGF-BB and PDGF-CC. Previous studies had revealed that olaratumab might exert anti- 
tumour activity in human sarcoma xenograft models (68). The results of the phase $\mathrm{Ib} / \mathrm{II}$ study, randomising 133 patients to receive olaratumab plus doxorubicin or doxorubicin alone, showed a median PFS of 6.6 months (95\% CI: 4.1-8.3 months) and 4.1 months (95\% CI: 2.8-5.4 months), and an objective RR of $18.2 \%$ (95\% CI: 9.8-29.6\%) and $11.9 \%$ (95\% CI: 5.3-22.2\%), respectively. The addition of olaratumab to doxorubicin indicated a much more improvement in OS, with a 11.8-month difference between these two arms. The median OS was 26.5 months (95\% CI: 20.9-31.7 months) and 14.7 months (95\% CI: 9.2-17.1 months) respectively. Based upon the improvement of OS, both FDA and EMA approved its use in the firstline setting in combination with doxorubicin. Until now, a confirmatory phase 3 study, the ANNOUNCE (NCT02451943), has finished enrollment.

\section{Immunotherapy: immune checkpoint inhibitors}

Immunotherapy has been one of the major breakthroughs in oncology. As for STS, the efficacy remains controversial. Of all the pathway discovered, PD-1/PD-L1 axis seems to attract the most attentions. Tumor PD-L1 expression has been reported in up to $65 \%$ of different subtypes of sarcomas and the degree of $\mathrm{PD}-1$ positivity in tumorinfiltrating lymphocytes (TILs) and PD-L1 expression in tumor specimens from 105 cases of STS, has been correlated with a poorer prognosis and more aggressive disease $(69,70)$. Nivolumab is a human IgG4 anti-PD-1 monoclonal antibody which has been found that it could exert an antitumor effect in metastatic sarcomas (71). In a retrospective study, a total of 28 patients with a metastatic or unresectable soft tissue or bone sarcoma received nivolumab $3 \mathrm{mg} / \mathrm{kg}$ IV every 2 weeks alone $(\mathrm{N}=10)$ or in combination with pazopanib $(\mathrm{N}=18)$. Among 24 evaluable patients, 12 patients had clinical benefit $(\mathrm{PR}+\mathrm{SD}), 12$ had progressive disease (PD). They observed three PR: one dedifferentiated chondrosarcoma, one epithelioid sarcoma and one maxillary osteosarcoma (last two patients on pazopanib); nine patients had SD including three LMS; 12 patients had progression of disease including 4 LMS (71).

Ipilimumab is a monoclonal antibody targeting CTLA-4 which seemed to have a very minimal effect when used alone against sarcomas. A multicenter, open-label, randomised, phase 2 study of nivolumab with or without ipilimumab aimed to investigate its activity and safety for the treatment of sarcoma. Enrolled patients were assigned (1:1) to nivolumab $3 \mathrm{mg} / \mathrm{kg}$ every 2 weeks, or nivolumab $3 \mathrm{mg} / \mathrm{kg}$ plus ipilimumab $1 \mathrm{mg} / \mathrm{kg}$ every 3 weeks for four doses. The primary endpoint was the proportion of patients achieving a confirmed objective response. Eight-five eligible patients were allocated to receive either nivolumab monotherapy (43 patients) or nivolumab plus ipilimumab (42 patients). The primary endpoint analysis was done in the first 76 eligible patients (38 patients per group). The number of confirmed responses was $2(5 \%)$ in the nivolumab group and $6(16 \%)$ in the nivolumab plus ipilimumab group. Serious treatment-related adverse events occurred in 8 (19\%) of 42 patients receiving monotherapy and 11 (26\%) of 42 patients receiving combination therapy. There were no treatmentrelated deaths. This trial indicated that the efficacy is much limited for nivolumab alone in an unselected sarcoma population, while nivolumab combined with ipilimumab demonstrated promising efficacy with a manageable toxicity in certain sarcoma subtypes, such as UPS, myxofibrosarcoma as well as LMS and angiosarcoma (72).

In the SARC028 phase 2 study, 40 patients with high grade, metastatic STS and 40 patients with bone sarcomas (osteosarcoma, Ewing's sarcoma, and dedifferentiated chondrosarcoma) were enrolled to receive pembrolizumab alone every three weeks until progression (73). Overall 11 of the 40 patients with STS had their tumors shrink while only three patients with bone sarcomas had tumor shrinkage. Eleven responding patients in the STS arm included four patients with UPS, 5 dedifferentiated liposarcoma (dLPS), 1 with synovial sarcoma and 1 with LMS. The patients with response in bone tumors included one patient with Ewing's sarcoma, one with osteosarcoma, and one with dedifferentiated chondrosarcoma. Pembrolizumab as a single agent showed activity in unselected sarcoma subtypes with an ORR of $17.5 \%$ and a $55 \% 3$-month PFS; UPS and DDLS were the histologies that seemed to benefit the most.

Compared with SARC028 study, another investigation enrolled 57advanced STS patients [15 LMS, 16 UPS, 10 gastrointestinal stromal tumors (GIST) and 16 others] who received $50 \mathrm{mg}$ twice daily cyclophosphamide 1 week on and 1 week off and $200 \mathrm{mg}$ of IV pembrolizumab every 3 weeks (74). The exploration indicated that 31 patients (10, LMS; 7, UPS; 8, others; and 6, GIST) with PD and 16 patients (3, LMS; 5, UPS; 5, others; and 3, GIST) with SD, 3 patients (1 patient with a STF, 1 with an endometrial stromal sarcoma, and 1 with a GIST) with progression free for 6 months. One objective response was observed in a patient with initially progressive SFT. The 6-months nonprogression rate was $0 \%, 0 \%, 14.3 \%$ and $11.1 \%$ for LMS, UPS, other and GIST, respectively. Further study found 
that the unique PR patient with SFT bearing more than $10 \%$ PD-L1-positive immune cells and the tumor had mild IDO-1 (indoleamine 2,3-dioxygenase 1) positive immune cells, a lower CD68 positive cell density and a higher CD8positive cell density. These findings may help us to further explore the mechanism of checkpoint inhibitors in STS.

The value of immunotherapy in STS is still largely unexplored. Further research focus on better patient selection and to investigate new combinatorial strategies.

\section{Summary}

Due to the advances of systemic treatment for STS in recent years, the outcome of STS has been greatly improved. As the molecular pathogenic basis of various histologic subtypes of STS has been revealed, the development of other promising molecular targeted therapy and immunotherapy will move the therapeutic modality from the all-fits-one approach for a more personalized therapeutic algorithm in the near future.

\section{Acknowledgements}

None.

\section{Footnote}

Conflicts of Interest: The authors have no conflicts of interest to declare.

\section{References}

1. Adjuvant chemotherapy for localised resectable softtissue sarcoma of adults: meta-analysis of individual data. Sarcoma Meta-analysis Collaboration. Lancet 1997;350:1647-54.

2. Pervaiz N, Colterjohn N, Farrokhyar F, et al. A systematic meta-analysis of randomized controlled trials of adjuvant chemotherapy for localized resectable soft-tissue sarcoma. Cancer 2008;113:573-81.

3. Frustaci S, Gherlinzoni F, De Paoli A, et al. Adjuvant chemotherapy for adult soft tissue sarcomas of the extremities and girdles: results of the Italian randomized cooperative trial. J Clin Oncol 2001;19:1238-47.

4. Woll PJ, Reichardt P, Le Cesne A, et al. Adjuvant chemotherapy with doxorubicin, ifosfamide, and lenograstim for resected soft-tissue sarcoma (EORTC 62931): a multicentre randomised controlled trial. Lancet
Oncol 2012;13:1045-54.

5. Bramwell V, Rouesse J, Steward W, et al. Adjuvant CYVADIC chemotherapy for adult soft tissue sarcoma-reduced local recurrence but no improvement in survival: a study of the European Organization for Research and Treatment of Cancer Soft Tissue and Bone Sarcoma Group. J Clin Oncol 1994;12:1137-49.

6. Coindre JM, Terrier P, Bui NB, et al. Prognostic factors in adult patients with locally controlled soft tissue sarcoma. A study of 546 patients from the French Federation of Cancer Centers Sarcoma Group. J Clin Oncol 1996;14:869-77.

7. Le Cesne A, Ouali M, Leahy MG, et al. Doxorubicinbased adjuvant chemotherapy in soft tissue sarcoma: pooled analysis of two STBSG-EORTC phase III clinical trials. Ann Oncol 2014;25:2425-32

8. Kattan MW, Leung DH, Brennan MF. Postoperative nomogram for 12-year sarcoma-specific death. J Clin Oncol 2002;20:791-6.

9. Callegaro D, Miceli R, Bonvalot S, et al. Development and external validation of two nomograms to predict overall survival and occurrence of distant metastases in adults after surgical resection of localised soft-tissue sarcomas of the extremities: a retrospective analysis. Lancet Oncol 2016;17:671-80.

10. Pasquali S, Colombo C, Pizzamiqlio S, et al. High-risk soft tissue sarcoma treated with perioperative chemotherapy: improving prognostic classification in a randomized clinical trial. Eur J Cancer 2018;93:28-36.

11. Gortzak E, Azzarelli A, Buesa J, et al. A randomised phase II study on neo-adjuvant chemotherapy for 'high-risk' adult soft-tissue sarcoma. Eur J Cancer 2001;37:1096-103.

12. Gronchi A, Frustaci S, Mercuri M, et al. Short, fulldose adjuvant chemotherapy in high-risk adult soft tissue sarcomas: a randomized clinical trial from the Italian Sarcoma Group and the Spanish Sarcoma Group. J Clin Oncol 2012;30:850-6.

13. Gronchi A, Stacchiotti S, Verderio P, et al. Short, full-dose adjuvant chemotherapy (CT) in high-risk adult soft tissue sarcomas (STS): long-term follow-up of a randomized clinical trial from the Italian Sarcoma Group and the Spanish Sarcoma Group. Ann Oncol 2016;27:2283-88.

14. Gronchi A, Ferrari S, Quagliuolo V, et al. Histotypetailored neoadjuvant chemotherapy versus standard chemotherapy in patients with high-risk soft-tissue sarcomas (ISG-STS 1001): an international, open-label, randomised, controlled, phase 3, multicentre trial. Lancet Oncol 2017;18:812-22. 
15. Casali PG, Abecassis N, Bauer S, et al. Soft tissue and visceral sarcomas: ESMO-EURACAN Clinical Practice Guidelines for diagnosis, treatment and follow-up. Ann Oncol 2018. [Epub ahead of print].

16. Judson I, Verweij J, Gelderblom H, et al. Doxorubicin alone versus intensified doxorubicin plus ifosfamide for first-line treatment of advanced or metastatic soft-tissue sarcoma: a randomised controlled phase 3 trial. Lancet Oncol 2014;15:415-23.

17. Seddon B, Strauss SJ, Whelan J, et al. Gemcitabine and docetaxel versus doxorubicin as first-line treatment in previously untreated advanced unresectable or metastatic soft-tissue sarcomas (GeDDiS): a randomised controlled phase 3 trial. Lancet Oncol 2017;18:1397-410.

18. Chawla SP, Papai Z, Mukhametshina G, et al. Firstline aldoxorubicin vs doxorubicin in metastatic or locally advanced unresectable soft-tissue sarcoma: a phase $2 \mathrm{~b}$ randomized Clinical Trial. JAMA Oncol 2015;1:1272-80.

19. Gupta S, Gouw L, Wright J, et al. Phase II study of amrubicin (SM-5887), a synthetic 9-aminoanthracycline, as first line treatment in patients with metastatic or unresectable soft tissue sarcoma: durable response in myxoid liposarcoma with TLS-CHOP translocation. Invest New Drugs 2016;34:243-52.

20. Tap WD, Papai Z, Van Tine BA, et al. Doxorubicin plus evofosfamide versus doxorubicin alone in locally advanced, unresectable or metastatic soft-tissue sarcoma (TH CR406/SARC021): an international, multicentre, open-label, randomised phase 3 trial. Lancet Oncol 2017;18:1089-103.

21. Cianfrocca M, Lee S, Von Roenn J, et al. Randomized trial of paclitaxel versus pegylated liposomal doxorubicin for advanced human immunodeficiency virus-associated Kaposi sarcoma: evidence of symptom palliation from chemotherapy. Cancer 2010;116:3969-77.

22. Schöffski P, Ray-Coquard IL, Cioffi A, et al. Activity of eribulin mesylate in patients with soft-tissue sarcoma: a phase 2 study in four independent histological subtypes. Lancet Oncol 2011;12:1045-52.

23. Eribulin shows promise in advanced sarcoma. Cancer Discov 2015;5:OF4.

24. D'Incalci M, Galmarini CM. A review of trabectedin (ET743): a unique mechanism of action. Mol Cancer Ther 2010;9:2157-63.

25. Martínez N, Sanchez-Beato M, Camero A, et al. Transcription signature of Ecteinascidin 743 in human sarcoma cell explanted from chemo-naïve patients. Mol Cancer Ther 2005;4:814-23.

26. Delaloge S, Yovine A, Taamma A, et al. Ecteinascidin-743: a marine-derived compound in advanced, pretreated sarcoma patients--preliminary evidence of activity. J Clin Oncol 2001;19:1248-55.

27. Taamma A, Misset JL, Riofrio M, et al. Phase I and pharmacokinetic study of ecteinascidin-743, a new marine compound, administered as a 24-hour continuous infusion in patients with solid tumors. J Clin Oncol 2001;19:1256-65.

28. Morgan JA, Le Cesne A, Chawla S, et al. Randomized phase II study of trabectedin in patients with liposarcoma and leiomyosarcoma after failure of prior anthracylines and ifosfamide. ASCO Meeting Abstracts, 2007;25:10060.

29. Yovine A, Riofrio M, Blay JY, et al. Phase II study of ecteinascidin-743 in advanced pretreated soft tissue sarcoma patients. J Clin Oncol 2004;22:890-9.

30. Garcia-Carbonero R, Supko JG, Manola J, et al. Phase II and pharmacokinetic study of ecteinascidin 743 in patients with progressive sarcomas of soft tissues refractory to chemotherapy. J Clin Oncol 2004;22:1480-90.

31. Le Cesne A, Blay JY, Judson I, et al. Phase II study of ET-743 in advanced soft tissue sarcomas: a European Organisation for the Research and Treatment of Cancer (EORTC) soft tissue and bone sarcoma group trial. J Clin Oncol 2005;23:576-84.

32. Huygh G, Clement PM, Dumez H, et al. Ecteinascidin-743: evidence of activity in advanced, pretreated soft tissue and bone sarcoma patients. Sarcoma 2006;2006:56282.

33. Monk BJ, Blessing JA, Street DG, et al. A phase II evaluation of trabectedin in the treatment of advanced, persistent, or recurrent uterine leiomyosarcoma: a gynecologic oncology group study. Gynecol Oncol 2012;124:48-52.

34. Gronchi A, Bui BN, Bonvalot S, et al. Phase II clinical trial of neoadjuvant trabectedin in patients with advanced localized myxoid liposarcoma. Ann Oncol 2012;23:771-6.

35. Grosso F, Jones RL, Demetri GD, et al. Efficacy of trabectedin (ecteinascidin-743) in advanced pretreated myxoid liposarcomas: a retrospective study. Lancet Oncol 2007; 8:595-602.

36. Sanfilippo R, Grosso F, Jones RL, et al. Trabectedin in advanced uterine leiomyosarcomas: a retrospective case series analysis from two reference centers. Gynecol Oncol 2011;123:553-6.

37. Demetri GD, von Mehren M, Jones RL. Efficacy and Safety of Trabectedin or Dacarbazine for Metastatic Liposarcoma or Leiomyosarcoma After Failure of Conventional Chemotherapy: Results of a Phase III 
Randomized Multicenter Clinical Trial. J Clin Oncol 2016;34:786-93.

38. Forni C, Minuzzo M, Virdis E, et al. Trabectedin (ET-743) promotes differentiation in myxoid liposarcoma tumors. Mol Cancer Ther 2009 8:449-57.

39. Sanfilippo R, Dileo P, Blay JY, et al. Trabectedin in advanced synovial sarcomas: a multicenter retrospective study from four European institutions and the Italian Rare Cancer Network. Anticancer Drugs 2015;26:678-81.

40. Cassier PA, Dufresne A, Blay JY, et al. Trabectedin and its potential in the treatment of soft tissue sarcoma. Ther Clin Risk Manag 2008;4:109-16.

41. Grosso F, Dileo P, Sanfilippo R, et al. Steroid premedication markedly reduces liver and bone marrow toxicity of trabectedin in advanced sarcoma. Eur J Cancer 2006;42:1484-90.

42. Takahashi N, Li WW, Banerjee D, et al. Sequencedependent enhancement of cytotoxicity produced by ecteinascidin 743 (ET-743) with doxorubicin or paclitaxel in soft tissue sarcoma cells. Clin Cancer Res 2001;7:3251-7.

43. Blay JY, von Mehren M, Samuels BL, et al. Phase I combination study of trabectedin and doxorubicin in patients with soft-tissue sarcoma. Clin Cancer Res 2008;14:6656-62.

44. Hensley ML, Maki R, Venkatraman E, et al. Gemcitabine and docetaxel in patients with unresectable leiomyosarcoma: results of a phase II trial. J Clin Oncol 2002;20:2824-31.

45. Sutton G, Blessing JA, Malfetano JH. Ifosfamide and doxorubicin in the treatment of advanced leiomyosarcomas of the uterus: a Gynecologic Oncology Group study. Gynecol Oncol 1996;62:226-9.

46. Pautier P, Floquet A, Chevreau C, et al. Trabectedin in combination with doxorubicin for first-line treatment of advanced uterine or soft-tissue leiomyosarcoma (LMS02): a non-randomised, multicentre, phase 2 trial. Lancet Oncol 2015;16:457-64.

47. Ryan CW, Merimsky O, Agulnik M, et al. PICASSO III: A Phase III, Placebo-Controlled Study of Doxorubicin With or Without Palifosfamide in Patients With Metastatic Soft Tissue Sarcoma. J Clin Oncol 2016;34:3898-905.

48. Woll PJ, Judson I, Lee SM, et al. Temozolomide in adult patients with advanced soft tissue sarcoma: a phase II study of the EORTC Soft Tissue and Bone Sarcoma Group. Eur J Cancer 1999;35:410-12.

49. Garcia del Muro X, Lopez-Pousa A, Martin J, et al. A phase II trial of temozolomide as a 6-week, continuous, oral schedule in patients with advanced soft tissue sarcoma: a study by the Spanish Group for Research on Sarcomas. Cancer 2005;104:1706-12.

50. Talbot SM, Keohan ML, Hesdorffer M, et al. A phase II trial of temozolomide in patients with unresectable or metastatic soft tissue sarcoma. Cancer 2003;98:1942-6.

51. Stacchiotti S, Tortoreto M, Bozzi F. Dacarbazine in solitary fibrous tumor: a case series analysis and preclinical evidence vis-a-vis temozolomide and antiangiogenics. Clin Cancer Res 2013;19:5192-201.

52. Park MS, Patel SR, Ludwiq JA, et al. Activity of temozolomide and bevacizumab in the treatment of locally advanced, recurrent, and metastatic hemangiopericytoma and malignant solitary fibrous tumor. Cancer 2011;117:4939-47.

53. Buesa JM, Mouridsen HT, van Oosterom AT, et al. Highdose DTIC in advanced soft-tissue sarcomas in the adult. A phase II study of the E.O.R.T.C. Soft Tissue and Bone Sarcoma Group. Ann Oncol 1991;2:307-9.

54. Borden EC, Amato DA, Rosenbaum C, et al. Randomized comparison of three adriamycin regimens for metastatic soft tissue sarcomas. J Clin Oncol 1987;5:840-50.

55. Sharma S, Takyar S, Manson SC, et al. Efficacy and safety of pharmacological interventions in second- or later-line treatment of patients with advanced soft tissue sarcoma: a systematic review. BMC Cancer 2013;13:385.

56. Ray-Coquard IL, Domont J, Tresch-Bruneel E, et al. Paclitaxel Given Once Per Week With or Without Bevacizumab in Patients With Advanced Angiosarcoma: A Randomized Phase II Trial. J Clin Oncol 2015;33:2797-802.

57. Martin-Broto J, Pousa AL, de Las Penas R, et al. Randomized Phase II Study of Trabectedin and Doxorubicin Compared With Doxorubicin Alone as FirstLine Treatment in Patients With Advanced Soft Tissue Sarcomas: A Spanish Group for Research on Sarcoma Study. J Clin Oncol 2016;34:2294-302.

58. Taylor BS, Barretina J, Maki RG, et al. Advances in sarcoma genomics and new therapeutic targets. Nat Rev Cancer 2011;11:541-57.

59. Sleijfer S, Ray-Coquard I, Papai Z, et al. Pazopanib, a multikinase angiogenesis inhibitor, in patients with relapsed or refractory advanced soft tissue sarcoma: a phase II study from the European organisation for research and treatment of cancer-soft tissue and bone sarcoma group (EORTC study 62043). J Clin Oncol 2009;27:3126-32

60. van der Graaf WT, Blay JY, Chawla SP, et al. Pazopanib for metastatic soft-tissue sarcoma (PALETTE): a 
randomised, double-blind, placebo-controlled phase 3 trial. Lancet 2012;379:1879-86.

61. Lin B, Song X, Yang D, et al. Anlotinib inhibits angiogenesis via suppressing the activation of VEGFR2, PDGFR beta and FGFR1. Gene 2018;654:77-86.

62. Xie C, Wan X, Quan H, et al. Preclinical characterization of anlotinib, a highly potent and selective vascular endothelial growth factor receptor-2 inhibitor. Cancer Sci 2018;109:1207-19

63. Chi Y, Yao Y, Wang S, et al. Anlotinib for metastasis soft tissue sarcoma: A randomized, double-blind, placebocontrolled and multi-centered clinical trial. J Clin Oncol 2018;36: abstr 11503.

64. Binh, MB, Sastre-Garau X, Guilou L, et al. MDM2 and CDK4 immunostainings are useful adjuncts in diagnosing well-differentiated and dedifferentiated liposarcoma subtypes: a comparative analysis of $559 \mathrm{soft}$ tissue neoplasms with genetic data. Am J Surg Pathol 2005;29:1340-7.

65. Dickson MA, Tap WD, Keohan ML, et al. Phase II trial of the CDK4 inhibitor PD0332991 in patients with advanced CDK4-amplified well-differentiated or dedifferentiated liposarcoma. J Clin Oncol 2013;31:2024-8.

66. Dickson MA, Schwartz GK, Keohan ML, et al.

Progression-free survival among patients with welldefferentiated or dedifferentiated liposarcoma treated with CDK4 inhibitor palbociclib: a phase 2 clinical trial. JAMA Oncol 2016;2:937-40.

67. Tap W.D, Jones RL, van Tine BA, et al. Olaratumab and doxorubicin versus doxorubicin alone for treatment of softtissue sarcoma: an open-label phase $1 \mathrm{~b}$ and randomised

Cite this article as: Liu W, Jiang Q, Zhou Y. Advances of systemic treatment for adult soft-tissue sarcoma. Chin Clin Oncol 2018;7(4):42. doi: 10.21037/cco.2018.08.02 phase 2 trial. Lancet 2016;388:488-97.

68. Loizos N, Xu Y, Huber J, et al. Targeting the plateletderived growth factor receptor alpha with a neutralizing human monoclonal antibody inhibits the growth of tumor xenografts: implications as a potential therapeutic target. Mol Cancer Ther 2005;4:369-79.

69. D'Angelo SP, Shoushtari AN, Agaram NP, et al. Prevalence of tumor-infiltrating lymphocytes and PD-L1 expression in the soft tissue sarcoma microenvironment. Hum Pathol 2015;46:357-65.

70. Kim JR, Moon YJ, Kwon KS, et al. Tumor infiltrating PD1-positive lymphocytes and the expression of PD-L1 predict poor prognosis of soft tissue sarcomas. PLoS One 2013;8:e82870.

71. Paoluzzi L, Cacavio A, Ghesani M, et al. Response to antiPD1 therapy with nivolumab in metastatic sarcomas. Clin Sarcoma Res 2016;6:24.

72. D'Angelo SP, Mahoney MR, Van Tine BA, et al. Nivolumab with or without ipilimumab treatment for metastatic sarcoma (Alliance A091401): two open-label, non-comparative, randomised, phase 2 trials. Lancet Oncol 2018;19:416-26.

73. Tawbi HAH, Burgess M, Crowley J, et al. Safety and efficacy of PD-1 blockade using pembrolizumab in patients with advanced soft tissue (STS) and bone sarcomas (BS): Results of SARC028-A multicenter phase II study. J Clin Oncol 2016;34:abstr 11006.

74. Toulmonde M, Penel N, Adam J, et al. Use of PD-1 Targeting, Macrophage Infiltration, and IDO Pathway Activation in Sarcomas: A Phase 2 Clinical Trial. JAMA Oncol 2018;4:93-7. 